\title{
INVESTIGACIONES
}

\section{Procrastinación, adicción al internet y rendimiento académico de estudiantes universitarios ecuatorianos}

\author{
Procastination, internet addiction, and academic performance \\ in Ecuadorian college students
}

\section{Carlos Ramos-Galarza ${ }^{a}$, Janio Jadán-Guerrero ${ }^{b}$, Lorena Paredes-Núñez ${ }^{c}$, Mónica Bolaños-Pasquel ${ }^{d}$,Antonio Gómez-García ${ }^{e}$}

\author{
${ }^{\text {a }}$ Departamento de Psicología Clínica, Universidad Internacional SEK del Ecuador, Quito, Ecuador. \\ Correo electrónico: carlos.ramos@uisek.edu.ec \\ ${ }^{\mathrm{b}}$ Centro de Investigación, Universidad Tecnológica Indoamérica, Quito, Ecuador. \\ Correo electrónico: janiojadan@uti.edu.ec \\ ${ }^{\text {c }}$ Departamento de Psicología Clínica, Universidad Internacional SEK del Ecuador, Quito, Ecuador. \\ Correo electrónico: lorenaparedes@uisek.edu.ec \\ ${ }^{\text {d }}$ Centro de Investigación en Psicología del Ecuador, Quito, Ecuador. \\ Correo electrónico: monicabolanos@gmail.com
}

${ }^{\text {e }}$ Centro de Investigación de Ciencias del Trabajo y Comportamiento Humano, Universidad Internacional SEK del Ecuador, Quito, Ecuador. Correo electrónico: antonio.gomez@uisek.edu.ec

\begin{abstract}
RESUMEN
Se reporta una investigación que analizó la relación entre la procrastinación, adicción al internet y rendimiento académico de universitarios. Se aplicó metodología mixta de dos fases. En la primera, se ejecutó un diseño cuantitativo, no experimental, transversal y alcance correlacional. Los resultados indican que, la procrastinación correlaciona con la adicción al internet $(r=0,50, p=<0,001)$ y que los estudiantes con alto rendimiento académico, presentan un menor comportamiento procrastinador $t_{(244)}=-3,60, \mathrm{p}<0,001$ y de adicción al internet $t_{(244)}=-2,44$, $\mathrm{p}=0,01$, a diferencia de universitarios con un rendimiento académico inferior. En la fase cualitativa se encontraron narrativas asociadas a la crianza recibida, experiencias pasadas determinantes, uso de funciones cognitivas, uso del internet y otras categorías. Finalmente, se discuten los datos en relación a investigaciones previas y mediante la integración de los resultados cuantitativos y cualitativos.
\end{abstract}

Palabras clave: aprendizaje, ciberespacio, creencias del estudiante, problemas de aprendizaje, Ecuador.

\begin{abstract}
This investigation analyzed the relationship between procrastination, internet addiction, and academic performance of college students. Mixed two-phase methodology was used. In the first phase, a quantitative, non-experimental, cross-sectional and correlational design was executed. The results indicate that procrastination correlates with internet addiction $(\mathrm{r}=0.50, \mathrm{p}=<0.001)$ and students with high academic performance have a lower incidence of procrastinating behavior $\mathrm{t}(244)=-3.60, \mathrm{p}<0.001$ and internet addiction $\mathrm{t}(244)=-2.44, \mathrm{p}=0.01$ compared to university students with lower academic performance. In the qualitative phase, narratives were found associated with parenting style experienced, past experiences, use of cognitive functions, use of the internet, and other categories. In conclusion, the data are discussed in relation to previous research and through the integration of quantitative and qualitative results.
\end{abstract}

Key words: learning, cyberspace, student beliefs, learning problems, Ecuador. 


\section{INTRODUCCIÓN}

El éxito académico del estudiante universitario tiene, en gran medida, relación con la consciencia que éste tiene de su accionar para resolver con éxito los diferentes retos que se van presentando dentro de su formación, por ejemplo, situaciones que demandan la aplicación de recursos comportamentales y cognitivos para cumplir de manera satisfactoria con la carga horaria, responsabilidades académicas, tareas, organización del estudio, evaluaciones, entre otros (Dominguez, Villegas \& Centeno, 2014).

$\mathrm{Si}$ bien, el ideal de estudiante universitario, sería aquel que cumple de manera satisfactoria con todas las demandas del medio educativo superior, ésta no es una realidad absoluta, ya que existe un elevado porcentaje de estudiantes que no son capaces de tener éxito académico, porque presentan, entre otros fenómenos, un comportamiento procrastinador (Wilson \& Bguyen, 2012).

El concepto procrastinación tiene que ver con un comportamiento caracterizado por la presencia de una postergación voluntaria de una actividad importante o con un propósito definido, a pesar de que, la no realización de sus responsabilidades pueda conllevar consecuencias negativas, incluso si las consecuencias positivas de realizar la tarea puedan ser más elevadas (Klingsieck, 2013; Mann, 2016).

Los estudiantes universitarios que postergan sus responsabilidades suelen caracterizarse por generar promesas de cumplimiento futuro de sus tareas, excusar y justificar su comportamiento de retraso en la presentación de trabajos, evadir la culpa de su no atención a las actividades que demandan un esfuerzo sostenido, evitar un cambio de paradigma para empoderarse de su comportamiento académico y por la tendencia a realizar actividades que tengan un refuerzo positivo inmediato y no de aquellas que demanden una proyección a un logro futuro (Ferrari, Johnson \& McCown, 1995; Quant \& Sánchez, 2012).

En diferentes estudios, se ha reportado que la procrastinación está asociada con diferentes factores, entre los más relevantes, condiciones de ansiedad ante el proceso académico y sus actividades de evaluación, bajos niveles de creencias de autoeficacia, tensión psicológica ante la pronta entrega de tareas académicas, bajo rendimiento académico, puntaje inferior en inventarios que valoran la inteligencia emocional, asociación a niveles socioeconómicos bajos y a una representación mental en donde, los estudiantes con mayores niveles de procrastinación atribuyen sus dificultades al poco o nulo interés que generan las actividades académicas y a sus limitadas habilidades de planificación del tiempo (Argumedo, Díaz \& Calderón, 2005; Chan, 2011; Clariana, Cladellas, Del Mar \& Gotzens, 2011; Jackson, Weiss, Lundquist \& Hooper, 2003; Klassen \& Kuzuku, 2009).

Como se lo ha descrito, son diversos los factores con los cuales se ha asociado a la procrastinación, en donde se pueden identificar diversos elementos que influyen en el comportamiento postergador de las responsabilidades del estudiante universitario. Uno de los que más influencia actualmente generan, es el contexto tecnológico de comunicación actual, que ha generado un profundo cambio en las prácticas de enseñanza y aprendizaje (Duart \& Lupiánez-Villanueva, 2005), en donde, estas condiciones que viven los universitarios, han generado un contexto que influye de manera significativa en la procrastinación, siendo el uso del internet el principal medio tecnológico en donde los universitarios consumen sin mayor beneficio la mayoría de su tiempo.

Según Contreras et al. (2011), en la web se ofrecen cientos de sitios donde el estudiante universitario realiza actividades placenteras sin generar mayor esfuerzo cognitivo y que le 
generan satisfacciones inmediatas. Esta situación ha sido reportada en investigación previa, en donde los estudiantes con mayores niveles de procrastinación, utilizan la mayoría de su tiempo libre en la navegación de redes sociales, chats, y otros lugares del cyber espacio que ofrecen actividades placenteras (Condori \& Mamani, 2016).

Parra et al. (2016) y García et al. (2008) describen que actualmente existe un uso y abuso del internet por parte de los jóvenes universitarios, ya que en este mismo contexto virtual,se encuentran plataformas virtuales de aprendizaje, donde el estudiante debe subir tareas e interactuar para aprender, sin embargo, a tan solo unos centímetros, se encuentran otras páginas, en donde el estudiante podría desperdiciar su tiempo con el uso de redes sociales, juegos en línea, reproductores de películas, entre otros recursos de la web, que se convierten en estímulos que aumentan el comportamiento procrastinador del estudiante universitario (Ndege et al., 2015).

Las investigaciones que han reportado la relación entre el uso excesivo de internet, la procrastinación y el rendimiento académico, al momento actual, no han sido concluyentes, por ejemplo, existen estudios que afirman la influencia positiva del uso del internet en el desempeño educativo, sin embargo, se han reportado estudios en donde se ha descrito que este comportamiento con la tecnología, influye negativamente en las calificaciones del estudiante (Raines, 2012; Torres-Díaz, Duart, Gómez-Alvarado, Marín-Gutiérrez \& Segarra-Faggioni, 2016).

Como proyección de dicha descripción, es importante continuar generando evidencia empírica que aporte en la comprensión de la relación entre las variables mencionadas. El realizar esta investigación tiene una gran importancia, puesto que en el contexto ecuatoriano alrededor de un 37,5\% de estudiantes universitarios pasan conectados más de seis horas al día a redes sociales como Facebook (Ramos \& Freire, 2016), lo cual nos invita a analizar si este tipo de prácticas pueden afectar en su rendimiento académico y analizar su relación con la procrastinación. Además, de una revisión previa, se ha identificado que no existen estudios sobre procrastinación y su relación con adicción al internet o al rendimiento académico dentro del contexto ecuatoriano, por lo que, esta investigación busca aportar evidencia empírica a esta línea de investigación que se encuentra todavía en desarrollo.

\section{METODOLOGÍA}

\subsection{DISEÑO DE INVESTIGACIÓN}

El diseño de investigación se basó en una metodología mixta de dos fases. En la primera, se aplicó un diseño de investigación cuantitativo no experimental, temporalidad transversal y alcance correlacional. En la segunda fase se aplicó en diseño de investigación cualitativo, basado en el paradigma constructivista, en donde las narrativas de los participantes fueron construyendo el fenómeno de estudio. Es importante mencionar que, esta segunda fase de la investigación, surgió para brindar una mayor riqueza en la interpretación de los resultados obtenidos en la fase cuantitativa y poder tener un entendimiento más profundo de las variables de estudio. 


\subsection{PARTICIPANTES}

En la fase cuantitativa participaron 250 estudiantes universitarios de Quito-Ecuador, 135 hombres (54\%) y 115 mujeres ( $46 \%)$, entre 17 y 31 años de edad $(M=21,17, D E=2,24)$. Los participantes fueron reclutados de las carreras de Psicología, Ingeniería industrial, Diseño gráfico, Arquitectura y Administración de Empresas, pertenecientes a dos universidades privadas de Quito, Ecuador. El nivel socio económico de los participantes fue medio y alto. El tipo de muestreo fue no probabilístico.

En la fase cualitativa se trabajó con 12 participantes, con quienes se realizó 4 entrevistas en profundidad ( 2 estudiantes con alto puntaje de procrastinación y 2 estudiantes con bajo puntaje de procrastinación) y dos focus group con 8 participantes (el primero con 4 estudiantes con ato puntaje de procrastinación y el segundo con 4 estudiantes con bajo puntaje de procrastinación).

\subsection{INSTRUMENTOS Y TÉCNICAS DE RECOLECCIÓN DE LA INFORMACIÓN}

Se utilizaron tres instrumentos. El primero fue la Escala de Adicción a Internet (EAIL) de Lam-Figueroa et al. (2011), que valora características sintomatológicas y disfuncionales relacionadas al excesivo uso del internet. Consta de once ítems que son valorados con un punto cuando la frecuencia del comportamiento es muy rara vez, dos puntos para rara vez, tres puntos para la opción a menudo y cuatro puntos para la opción siempre. En investigación previa se ha reportado un coeficiente de consistencia interna de la escala de $\alpha=0,84$ (LamFigueroa et al., 2011). Los siguientes son ejemplos de los ítems de la escala: Cuando no estoy conectado, imagino cómo será la próxima vez que estaré conectado a Internet, Dedico más tiempo a Internet del que pretendía, He llegado a perder clases por estar en Internet, etc.

El segundo instrumento fue la Escala de Procrastinación de Álvarez (2010), que valora la tendencia de los estudiantes a posponer o retrasar la finalización de sus responsabilidades. Está compuesto por trece ítems que son valorados en una escala, donde se califica con un punto cuando la respuesta es siempre, dos puntos cuando es casi siempre, tres puntos para a veces, cuatro puntos para pocas veces y cinco puntos para nunca. En evidencia empírica previa se ha reportado una consistencia interna de la escala de $\alpha=0,86$. Los siguientes son ejemplos de ítems de la escala: encuentro una excusa para no hacer lo que tenía que hacer, tiendo a perder mucho el tiempo, me disgusta seguir pautas estrictas, etc.

El tercer instrumento fue un cuestionario de variables sociodemográficas donde se cuantificó la calificación de graduación de la secundaria, calificación de promedio en el semestre actual y su valoración sobre su rendimiento académico en las opciones alto, medio y bajo.

En la fase cualitativa se utilizó una entrevista en profundidad que sirvió como guía en la interacción que se tuvo con los participantes al momento de dialogar sobre la procrastinación, adicción al internet y el rendimiento académico en el contexto universitario. De igual manera, para realizar el focus group se utilizó una guía de preguntas que permitieron direccionar el diálogo con los participantes.

\subsection{PLAN DE ANÁLISIS DE DATOS}

En la fase cuantitativa se utilizó estadística descriptiva de tendencia central y dispersión. Para analizar la relación entre las variables se utilizó correlación de Pearson. Para comparar 
las medias de las variables, se utilizó el proceso de $t$ de Student. Se aplicaron las pruebas de normalidad respectivas para el uso de los procesos paramétricos mencionados. Todos los análisis se los realizó en el paquete estadístico SPSS versión 23.

En la fase cualitativa se utilizó el análisis del contenido y codificación abierta para construir la teoría sustantiva que emergió en la interacción de los participantes en las entrevistas en profundidad y focus group. Este análisis se lo realizó en el software para análisis cualitativo Atlas.ti versión 7.

\subsection{PROCEDIMIENTO}

Se inició reclutando a los participantes de las instituciones de educación superior a las que pertenecían. Posteriormente, se solicitó su colaboración en la investigación y se firmaron los respectivos documentos de consentimiento informado. Una vez que se tuvo los instrumentos listos y completados se procedió al análisis cuantitativo. En la fase cualitativa se invitó a participantes con altos y bajos niveles de procrastinación identificados en la primera fase. Una vez que se tuvo el contenido lingüístico se procedió a su análisis. Es importante resaltar que, en todo momento de la presente investigación se cumplió con los estándares de ética para la investigación con seres humanos declarados en Helsinki (Williams, 2008).

\section{RESULTADOS}

\subsection{FASE CUANTITATIVA}

El primer análisis realizado fue para valorar la consistencia interna de las escalas utilizadas. La EAIL presentó como coeficiente de Alfa de Cronbach $\alpha=0,86$. La correlación entre los ítems y el total de la escala se encontró entre 0,40 y 0,64. No fue necesario eliminar algún ítem para que mejore el coeficiente de la escala.

La escala de procrastinación presentó un coeficiente de consistencia interna de $\alpha=0,90$. La correlación entre cada ítem con el total de la escala fue entre 0,55 y 0,72. Se decidió no considerar los ítems 5 y 13 de la escala porque disminuían la carga de su consistencia interna. Por tanto, se trabajo con los 11 ítems restantes que presentaron adecuados coeficientes de confiabilidad.

En la tabla 1 se presentan las medidas descriptivas obtenidas por los participantes.

Tabla 1. Estadísticas descriptivos de las variables en estudio

\begin{tabular}{|l|c|c|c|c|}
\hline Variable & $\mathrm{Mn}$ & $\mathrm{Mx}$ & $\mathrm{M}$ & $\mathrm{DE}$ \\
\hline Promedio Actual & 5,60 & 10,00 & 8,35 & 0,69 \\
\hline Adicción a Internet & 11 & 39 & 20,29 & 6,02 \\
\hline Procrastinación & 11,00 & 54,00 & 29,73 & 8,78 \\
\hline
\end{tabular}

Clave. Mn: mínimo, Mx: máximo, M: media y DE: desviación estándar. 


\section{Correlación entre las variables de estudio}

La procrastinación correlacionó significativamente con adicción al internet $(r=0,50$, $p=<0,001$ ), en donde se encontró una relación directamente proporcional, en donde, cuando la variable procrastinación asciende, también lo hace la adicción al internet.

\subsection{COMPARACIÓN SEGÚN RENDIMIENTO ACADÉMICO}

En el cuestionario de variables socio-demográficas se pidió a los estudiantes que valoraran su rendimiento académico. Se encontró que, 76 estudiantes seleccionaron al grupo de alto rendimiento, 170 al grupo de rendimiento medio y 4 estudiantes al grupo de bajo rendimiento. Se decidió comparar la procrastinación y adicción al internet, considerando como factores en la prueba $t$ de Student a los dos grupos de rendimiento alto y medio. No se incluyó al grupo de bajo rendimiento por sólo tener 4 estudiantes. En la comparación se encontró que, existen diferencias estadísticamente significativas en procrastinación $t_{(244)}=-$ $3,60, \mathrm{p}<0,001$ y en adicción a internet $t_{(244)}=-2,44, \mathrm{p}=0,01$; siendo los estudiantes con alto rendimiento quienes presentan menor procrastinación y menor adicción al internet, en comparación al grupo de rendimiento medio (ver Figura 1).

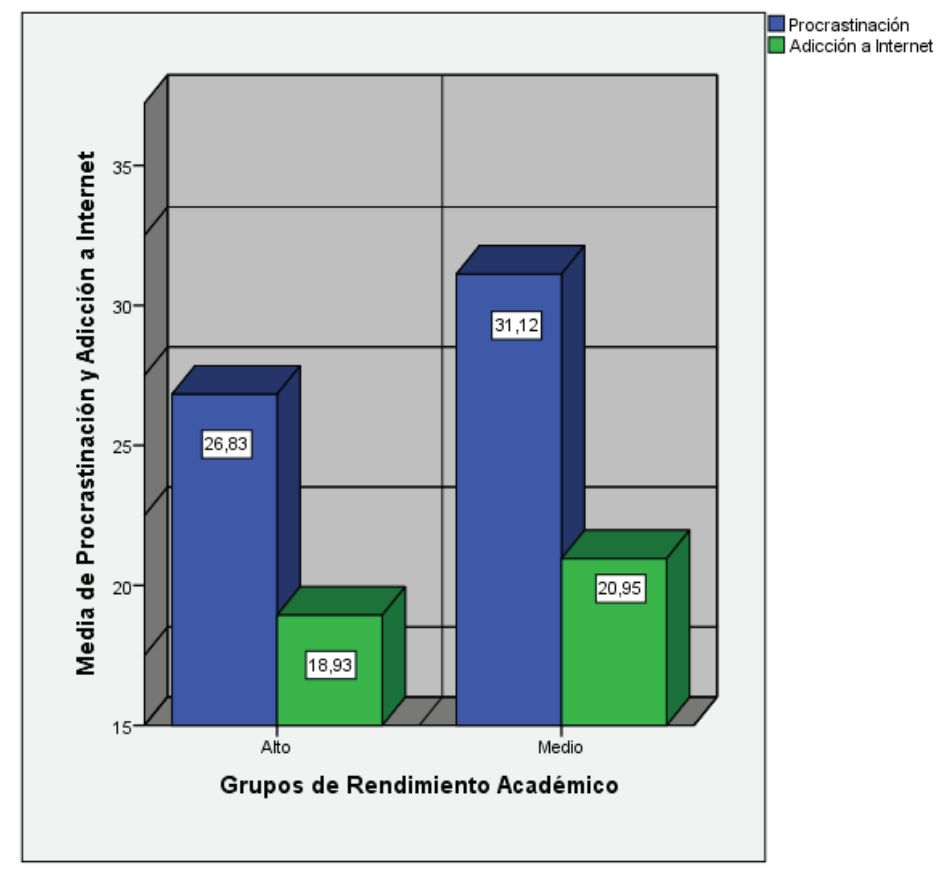

Figura 1. Representación gráfica de la media de procrastinación y adicción a internet, considerando como grupos de comparación el rendimiento académico alto y medio. Es importante que se considere que en adicción a internet y procrastinación, el puntaje mayor indica mayor problemática en la variable. 


\subsection{DIFERENCIAS ENTRE HOMBRES Y MUJERES}

Se procedió a comparar las variables analizadas, considerando como factores el género de los participantes, donde se encontró que, adicción a internet $t_{(248)}=-0,10, p=0,92$, procrastinación $t_{(248)}=-0,34, p=0,73$ y rendimiento académico $t_{(248)}=-0,48, p=0,62$ no presentan diferencias estadísticamente significativas.

\subsection{FASE CUALITATIVA}

Una vez se obtuvieron los datos cuantitativos se observó una diferencia entre los estudiantes, según sea su rendimiento académico, sin embargo, el cerrar la presente investigación en dicho punto dejaría inconclusa la comprensión de las variables de estudio. Por tal razón se decidió continuar con una segunda fase de estudio, para poder lograr una interpretación más profunda de los resultados.

A continuación, se presenta la teoría sustantiva que emergió en la interacción con los participantes en las entrevistas en profundidad y los focus group. Es importante mencionar al lector que, en cada categoría se realiza una interpretación y síntesis de los discursos de los participantes y se presentan citas textuales de las narrativas con mayor significado identificadas en la interacción lingüística.

\subsubsection{La crianza influye en la procrastinación}

En las entrevistas en profundidad se encontró que, los estudiantes con alto rendimiento son conscientes de que existen factores que han influido en su actual comportamiento para aprender, por ejemplo, manifiestan recuerdos de su experiencia infantil, mencionando afirmaciones como "a mí me criaron para ser autónoma y responsable con mis tareas", en donde se puede ver que, desde pequeños los estudiantes con alto rendimiento y bajo nivel de procrastinación, ha sido formado para actuar de manera adecuada al momento de aprender.

En cambio, si no existe una crianza orientada a motivar a que el niño sea consciente de sus responsabilidades, es probable que en la juventud, como se da en el caso de los estudiantes universitarios con altos niveles de procrastinación, no se desarrolle una adecuada consciencia de las consecuencias de sus actos y dependan en gran medida de sus padres para solucionar los conflictos a los que se enfrenten. En tal sentido, una de las participantes afirmó:

"Una compañera nuestra se retiró de la carrera porque no le gustaba, luego fue a otra carrera y tampoco le gustaba y tuvo una tercera experiencia donde le fue igual, el problema es que es mimada (hijo que ha sido criado en una sobreprotección por parte de los padres) y sus papás le consienten en todos sus caprichos (..) ella no siente la necesidad de ser autónoma"

Un participante afirmó que en su caso, sus padres los criaron de manera diferente a él y a su hermano, ya que a éste último no se lo reprendía cuando hacía alguna travesura o cometía algún error, en cambio, a él sí, lo cual influyó en su forma de ser actual, ya que él, actualmente, realiza sus actividades de forma independiente, mientras que, a su hermano (ambos son universitarios) es necesario que los padres supervisen cada paso que recorre. 
Otra participante habló de la lealtad al sacrificio que hicieron sus padres por poder brindarle su educación, en donde, lo único que siempre pudo hacer para contribuir a su familia es estudiar, “desde pequeña vi que mi papá hacía hasta lo imposible para educarnos, entonces, a mí no me quedaba de otra que cumplir con mis tareas, no podía dar más problemas en mi casa de los que ya existían".

Por otro lado, en la narrativa se identificó un elemento protector para que un estudiante aprenda a controlar el comportamiento procrastinador, el cual es, que desde pequeño aprenda normas y reglas de comportamiento para aprender, en donde tenga claras las consecuencias de presentar conductas negativas en su proceso de aprendizaje, como lo es la procrastinación: "Yo desde niña no podía distraerme en actividades que no sean mis tareas, porque mi papá era claro en las órdenes y yo tenía que obedecer (...) pobre de mí que no le haga caso"

\subsubsection{Sólo reviso mis novedades en internet... antes de empezar mis tareas}

En el diálogo con los estudiantes con altos niveles de procrastinación se identificó un comportamiento habitual al momento de iniciar sus tareas académicas, en donde, en primer lugar manifiestan revisar y comentar las novedades que se publican en la página de Facebook, Twitter y otras; luego responden a los mensajes recibidos en sus redes sociales, revisan constantemente su celular para ver los mensajes recibidos en WhatsApp, responden correos electrónicos, participan en alguna sesión rápida de algún video juego en línea y, luego de dicho tiempo de navegación virtual, realizan su tarea, la cual consiste en descargar algún documento de internet y adaptarlo en su forma para presentarlo, o en muchos casos, ni siquiera hacen trabajo alguno, porque les cuesta dejar de interactuar en los diferentes espacios propuestos en internet y pasar a cumplir con sus responsabilidades.

Cuando enciendo el computador para hacer alguna tarea, primero hablo con mis amigos, veo las fotos que publican, veo mis puntajes en los juegos de Facebook, chateo (...) reviso el resto de mis redes sociales, miro algún video musical de mis artistas favoritos, lo comparto con mis contactos (...).

Como se puede observar, el estudiante con altos niveles de procrastinación relata con detalles su experiencia diaria en internet, sin embargo, no manifiesta estrategias de aprendizaje que podría utilizar para cumplir con éxito un aprendizaje significativo, ya que la mayoría de su tiempo lo utiliza para disfrutar en los espacios que brinda el internet.

Además, al momento de realizar la discusión sobre esta categoría, surgió un fenómeno psicológico, en donde los participantes tomaron consciencia en el sentido de que, al iniciar sus tareas revisando sus redes sociales, utilizan sus recursos cognitivos para resolver esos problemas simples, lo que genera que, cuando empiezan con sus tareas ya se encuentran cansados, fatigados, o al menos, no en su $100 \%$ de rendimiento, ya que los primeros recursos cognitivos (los más valiosos) fueron utilizados en acciones procrastinadoras.

Hay algo que recién me doy cuenta al hablar aquí en grupo sobre este tema, que cuando uno primero revisa el Facebook o Instagram por varias horas, se cansa haciendo esas cosas y cuando empieza hacer los deberes de la universidad, es como que viene el sueño más rápido y uno ya no lo hace, muchas veces me quedo dormido, y eso se vuelve un hábito. 


\subsubsection{No todo en internet es malo}

Varios estudiantes con alto rendimiento académico y bajos niveles de procrastinación manifestaron que el pasar tiempo navegando en internet no es tan malo como se creería, ya que sí existen lugares en los cuales se puede aprender y actualizarse. Sin embargo, las horas invertidas en la navegación por la web por este grupo de estudiantes, no es para realizar actividades poco demandantes a nivel cognitivo o de distracción, sino que, sus grupos de interacción de Facebook tienen como finalidad el intercambio de conocimientos de su profesión, diarios digitales de noticias nacionales e internacionales, revistas científicas, grupos de discusión de temas sociales y demás espacios en los cuales pueden crecer a nivel intelectual y académico.

Yo utilizó el Facebook o Twitter para estar al tanto de las noticias del país y del mundo, casi no lo utilizó para ver las fotografías de mis contactos o comentar, yo suelo desactivar esas notificaciones, el chat casi ni lo utilizo (...) en los grupos a los que pertenezco debatimos temas relacionados con lo que vemos en la universidad, se comparte material de estudio y a veces nos apoyamos en la resolución de los ejercicios más difíciles que envían los ingenieros.

Un aspecto que se debe considerar, es que si bien, el internet es un factor que puede ser contraproducente para el cumplimiento de las múltiples responsabilidades de los universitarios, si es bien orientado podría convertirse en una herramienta de mucha utilidad en la construcción de conocimientos de los universitarios.

\subsubsection{Las funciones cognitivas de alto nivel}

En los estudiantes de alto rendimiento que indican niveles bajos de procrastinación, se puede identificar que, dentro de sus fortalezas al momento de aprender, utilizan funciones cognitivas de alta complejidad, como lo son la planificación, monitorización, verificación, organización de materiales e iniciativa.

Por ejemplo, en sus narrativas se identifican aspectos como la planificación, "el día anterior a la clase estudio algo de su contenido y reviso que lleve todo lo necesario". Monitorización de su aprendizaje "cuando me doy cuenta que estoy dejando de cumplir con mis responsabilidades de la universidad, enseguida vuelvo a mis tareas", "en mi casa yo siempre tuve claro los horarios, podía estar disfrutando con mis amigas y si ya llegaba la hora de entrar a hacer mis deberes tenía que hacerlo (...) porque así debe ser". En iniciativa manifiestan expresiones como "cuando el profesor explica algo en la clase, tomo apuntes y en mi casa profundizo en el tema, no me importa que no haya mandado la actividad como una tarea".

En la verificación, en su discurso se encuentran narrativas como "antes de presentar un trabajo leo que no tenga errores, porque si sólo lo bajas de internet y lo presentas no estás aprendiendo nada". En organización de materiales se observa que el estudiante con baja procrastinación cuenta con los elementos adecuados, dispuestos a su alcance para cumplir con éxito sus actividades, tal es el caso de varios participantes con dicha característica, que al iniciar el diálogo tenían a su alcance cuadernos de apuntes, resaltadores para identificar ideas principales y secundarias, papeles adhesivos para tomar notas, en fin, los 
recursos necesarios para acceder a los conocimientos de forma adecuada, a diferencia de los estudiantes del grupo de alto puntaje de procrastinación, quienes el único elemento que tenían dispuesto para la actividad, era su teléfono celular, el que por cierto, tuvo que pedirse que sea guardado porque les generaba distracción.

\subsubsection{El tiempo dedicado a las tareas}

Una estudiante de alto rendimiento manifiesta que es posible dejar de lado la conducta procrastinadora mediante un trabajo esforzado y sostenido, donde manifiesta "Yo prefiero hacer una tarea de forma seguida sin parar (...), podría trabajar sin problemas siete horas o hasta terminar". Como se puede observar, el estudiante con bajos niveles de procrastinación es capaz de mantener y regular sus procesos atencionales en una actividad demandante de un trabajo prolongado.

Esta situación no se presentaría en los estudiantes con altos niveles de procrastinación, quienes tienen dificultades en terminar las tareas que inician, se distraen con facilidad a medida que van realizando el trabajo o simplemente, lo abandonan.

A veces inicio un trabajo de la $\mathrm{U}$ y a los cinco minutos ya estoy revisando mi Facebook, luego ni me acuerdo de volver al trabajo (...), luego no me queda de otra que pedir a alguien que me lo envíe, hago un par de cambios y lo presento.

\subsubsection{Un cambio en el comportamiento procrastinador mejora el desempeño académico}

Varios estudiantes con bajos niveles de procrastinación, manifiestan que no siempre fueron capaces de controlar su comportamiento en el aprendizaje y realizar primero sus responsabilidades académicas y luego las de recreación.

Yo tomé la decisión de cerrar mi Facebook porque pasaba muchísimo tiempo conectada chateando, revisando las fotos de mis amigos (...) y empecé a dejar de lado el estudio, como casi pierdo mi beca por bajar en notas, no me quedó de otra que cerrar mis cuentas, concentrarme en mis responsabilidades y ahora me distraigo menos.

Como se puede observar, es posible que un estudiante pueda tomar conciencia de su comportamiento y realice los cambios necesarios para cumplir con las tareas académicas, sin embargo, no en todos los estudiantes es posible que realicen el acto de disminuir la procrastinación mediante la buena administración de su tiempo en las redes sociales, "en mi caso, yo cierro mi cuenta de Facebook y al otro día ya la abro y la uso con más fuerza".

Otros estudiantes narran que, cuando olvidan el celular en su casa se convierte en una situación que les genera mucha preocupación porque no saben quienes están escribiéndoles, que publican sus contactos, en fin, que está sucediendo en el mundo virtual. "Yo cuando olvido mi celular, no me importa perder la primera hora de clase, regreso a mi casa a buscarlo", "le llamo a mi mamá para que me lo traiga (celular), como ella me entiende lo que es estar sin celular, viene a dejármelo". 


\subsubsection{El evento que lo cambió todo}

Si bien, se ha identificado una condición en la cual, ciertos estudiantes toman conciencia y cambian su forma de actuar ante las responsabilidades académicas, ésta no se presentaría sola o de manera aislada, ya que, como se identificó en la narrativa de un párrafo anterior, una estudiante vivió una situación amenazante (perder su beca) que le obligó a tomar la decisión de cambiar su comportamiento, a diferencia de la participante que manifestó serle imposible cerrar su cuenta de Facebook y modificar su comportamiento procrastinador.

Otra participante narró un evento que afirma haber sido determinante en su vida y que siempre evoca en su mente cuando debe cumplir con una responsabilidad.

Recuerdo que, cuando era niña tenía que hacer deberes de la escuela y en mi casa no teníamos dinero, y recuerdo bien que mi papá tuvo que vender su reloj y su ropa para comprar leche para mi hermano (...), desde ese día supe que debía ser responsable y siempre que tengo que cumplir con mis responsabilidades académicas, viene a mi mente esa imagen de mi padre, no lo puedo defraudar.

\section{DISCUSIÓN Y CONCLUSIONES}

En el presente artículo se ha analizado la relación existente entre la procrastinación, rendimiento académico y adicción a internet de una muestra de estudiantes universitarios de Quito, Ecuador. Para cumplir con este fin, se ha diseñado un estudio con metodología mixta de dos fases.

En la primera fase, con un diseño cuantitativo, se encontró una correlación directamente proporcional entre procrastinación y adicción a internet. Esta afirmación sugiere que, los estudiantes con mayores niveles de procrastinación, pueden estar conectados por largas horas en sitios web y tienen una mayor probabilidad de realizar actividades de internet que presenten una gratificación inmediata, sin una demanda de esfuerzo cognitivo que, en términos generales, no generan mayor beneficio para su formación profesional y que influyen negativamente al momento en que deben cumplir con sus responsabilidades académicas.

Dentro de dicha afirmación, se ha reportado que los universitarios que presentan procrastinación tienden a utilizar grandes cantidades de tiempo en revisar sus bandejas de entrada de sus aplicaciones para recibir comunicaciones, a pesar de no haber sido alertados por el mensaje de alerta de llegada de algún correo o mensaje, en muchas ocasiones, lo hacen de manera automática, como una conducta motora condicionada con su celular o computador.

Desde la neurociencia se puede brindar una explicación que puede dar luces para entender el comportamiento procrastinador del universitario en el internet, ya que, según teorías del funcionamiento neurobiológico cerebral, se afirma que, cada vez que el universitario recibe un like por alguna publicación, notificaciones de visitas de tu perfil, se recibe o se envía mensajes, entre otras formas de gratificación inmediata, en el cerebro se segrega dopamina, neurotransmisor que brinda placer, satisfacción y que se asocia a estados de emoción positiva. Por esta razón, los universitarios con niveles elevados de procrastinación pasan largo tiempo buscando esta fuente de excitación, que aumenta las probabilidades de presentar una adicción al internet (Universidad de Navarra, 2012). 
En la comparación de la procrastinación y adicción a internet, considerando el rendimiento académico alto y medio de estudiantes como factores independientes, se encontró que, los estudiantes con alto rendimiento presentan niveles inferiores de procrastinación y adicción a internet. Este resultado sugiere que las dos variables estarían asociadas al nivel del rendimiento académico de los universitarios, lo cual es concordante con investigaciones previas que han descrito dicho hallazgo (Ackerman \& Gross, 2005; Klassen, Krawchuk, \& Rajani, 2008).

Al comparar la procrastinación y adicción a internet, según el género de los participantes, se encontró que no existen diferencias estadísticamente significativas, por lo que, las características en el comportamiento de postergación de las responsabilidades académicas y el uso excesivo del internet es similar en hombres y mujeres. Este resultado es concordante con un estudio previo realizado en Ecuador por Torres-Díaz et al. (2016).

Una vez realizado el análisis cuantitativo, se tomó la decisión de realizar una siguiente fase con una metodología de investigación cualitativa, basada en la entrevista en profundidad y focus group. La teoría sustantiva que emergió en las narrativas con los estudiantes, nos permitió profundizar e interpretar de una forma más completa los aspectos de interés en la investigación.

En primer lugar, se identificó que los estudiantes universitarios atribuyen la condición procrastinadora como una condición de efecto del estilo de crianza que han recibido, en donde, los estudiantes con padres mayormente permisivos podrían ser un factor que está implicado en el desarrollo de este comportamiento al momento de aprender. Mientras que, los universitarios con bajos niveles de procrastinación, narran experiencias de su crianza, en donde fueron formados para ser individuos autónomos y conscientes de las consecuencias de sus actos al momento de aprender.

Otro significado que emergió en la interacción y que permite interpretar con mayor riqueza los resultados de la fase cuantitativa, es la condición procrastinadora y de uso del internet que se expresó por los participantes con altos niveles de procrastinación, en donde se identificó que, el uso excesivo de las diferentes aplicaciones y recursos de la web, en donde pasan la mayoría de su tiempo, hacen que cumplan de manera menos eficiente con sus responsabilidades académicas o en el peor de los casos, no las cumplan.

Fue interesante interpretar este no avance a cumplir con las responsabilidades por el uso inicial de las redes sociales, juegos, páginas web, entre otros, que podría generar que el estudiante universitario no cumpla con sus tareas, ya que usa sus principales recursos cognitivos para iniciar con la navegación web y al momento de realizar su tarea llega fatigado cognitivamente y aumenta la probabilidad de cumplirla de manera deficiente o simplemente no hacerlo, ya que el proceso cognitivo atencional es limitado y sus mejor desempeño se lo encontrará al iniciar una actividad (Portellano, 2005).

A pesar de que, se encontró que el uso de internet es un factor en contra del adecuado aprendizaje del universitario, en el grupo de estudiantes con bajos niveles de procrastinación y alto rendimiento académico, se observó una situación diferente, ya que este tipo de estudiantes realizan un uso optimizado del internet, en donde, existe un control del tiempo destinado a esta actividad y una selección de sitios web que sean en favor de su aprendizaje. Este resultado sugiere que el uso del internet podría ser un factor a favor o en contra del desempeño académico universitario (Raines, 2012; Torres-Díaz et al., 2016), donde todo dependerá del control consciente y los objetivos que se desprendan de su uso.

En los universitarios con bajo nivel de procrastinación se identificó el uso de funciones cognitivas de alto nivel (Ramos \& Pérez-Salas, 2015) en su comportamiento diario para 
aprender. Lo cual lo convierte en un fctor protector (Cova, 2004), que disminuiría las conductas de postergación voluntaria de las responsabilidades académicas. A diferencia de los estudiantes altamente procrastinadores, en quienes no se identificó en su discurso algún aspecto que hiciera mención a la aplicación de funciones congitivas para regular su comportamiento al momento de aprender.

Por otro lado, se observó que en los estudiantes que han logrado superar la tendencia a manifestar un comportamiento procrastinador, narran en sus expresiones, un evento que les marcó la vida y les motivó a cambiar su comportamiento, tal es el caso, de situaciones infantiles o actúales universitarias, que les invitó a tomar conciencia de su capacidad para controlar su comportamiento y orientarlo hacia la consecución de objetivos de aprendizaje.

Como limitaciones del estudio, se debe mencionar que, en la etapa cuantitativa se utilizaron escalas de auto-reporte, lo cual podría estar influenciado por la subjetividad del estudiante al momento de reportar su comportamiento. Además, en la primera fase del estudio se trabajó con una muestra de universitarios pertenecientes a una ciudad determinada de Ecuador, lo que invita a ser prudentes al momento de realizar generalizaciones de los resultados descritos en el presente artículo.

En la fase cualitativa, se debe mencionar una limitación que es propia del método cualitativo, que es la carga subjetiva en la interpretación personal de los investigadores sobre el material lingüístico analizado. Lo cual no invalida sus resultados, ya que se los comprende dentro de un marco de referencia constructivista (Ramos, 2015), en donde la perspectiva de los participantes y de los investigadores es válida en la construcción de un fenómeno, no obstante, es menester tener presente esta afirmación al momento de interpretar los datos de la investigación.

Los resultados que se han descrito en el presente trabajo, invita a que los docentes del sistema educativo superior, consideren las dos variables estudiadas como factores que pueden influir en el rendimiento académico y, al igual que Xu (2016), consideramos que la intervención en la procrastinación es una responsabilidad conjunta del profesor y del estudiante, por lo que, se deben crear programas que beneficien a los universitarios que se ven afectados por la procrastinación y la adicción al internet.

En tal sentido, la investigación futura de las variables tratadas se proyecta a la ejecución de estudios con diseños experimentales, que nos permitan contrastar el efecto de una intervención entre estudiantes con procrastinación y adicción a internet y estudiantes controles que no presenten estos comportamientos. También, es de interés replicar el presente estudio a nivel nacional y con una muestra representativa de Ecuador.

\section{REFERENCIAS BIBLIOGRÁFICAS}

Ackerman, D. \& Gross, B. (2005). My instructur made me do it: Task characteristics of procrastination. Journal of Marketing Education, 27, 5-13.

Álvarez, O. (2010). Procrastinación general y académica en una muestra de estudiantes de secundaria de Lima metropolitana. Persona, 13, 159-177.

Argumedo, D., Díaz, K. \& Calderon, A. (2005). Evaluación de la confiabilidad y la estructura familiar de tres escalas de procrastinación crónica. Revista de la PUCP, 23 (1), 113-138.

Chan, L. (2011). Procrastinación académica como predictor en el rendimiento académico en jóvenes de educación superior. Temática Psicológica, 7 (1), 53-62. 
Clariana, M., Cladellas, R., Del Mar, M. \& Gotzens, C. (2011). La influencia del género en variables de la personalidad que condicionan el aprendizaje: inteligencia emocional y procrastinación académica. Revista Electrónica Interuniversitaria de Formación del Profesorado, 14 (3), 87-96.

Condori, Y. \& Mamani, K. (2016). Adicción al Facebook y procrastinación académica en estudiantes de la facultad de ingeniería y arquitectura de la Universidad Peruana Unión. Juliaca, Perú: (Tesis de Grado de Psicología).

Contreras, H., Mori, E., Lam, N., Gil, E., Hinostroza, W., Rojas, D., et al. (2011). Procrastinación en el estudio: exploración del fenómeno en adolescentes escolarizados. Revista Peruana de Epidemiología, 15 (3), 1-5.

Cova, F. (2004). La Psicopatología Evolutiva y los Factores de Riesgo y Protección: el Desarrollo de una Mirada Procesual. Revista de Psicología, 8 (1), 93-101.

Dominguez, S., Villegas, G. \& Centeno, S. (2014). Procrastinación académica: validación de una escala en una muestra de estudiantes de una universidad privada. Liberabit, 20 (2), 293-304.

Duart, J. \& Lupiánez-Villanueva, F. (2005). E-strategies in the Introduction and Use of Information and Comunication Technologies in the University. Universities and Knowledge Society Journal, 2 (1), 1-26. doi http://doi.org/10.7238/rusc.v2i1.243.

Ferrari, J.; Johnson, J. \& Mc Cown, W. (1995). Procrastination and task avoidance: theory, research and treatment. New York: Plenum.

García, J., Terol, M., Nieto, M., Lledó, A., Sánchez, S., Martín-Aragón, M. \& Sitges, E. (2008). El uso y abuso de Internet en jóvenes universitarios. Adicciones, 20 (2), 131-142.

Jackson, T.; Weiss, K.; Lundquist, J. \& Hooper, D. (2003). The impact of hope, procrastination and social activity on academic performance of mid western college students. Education, 124 (2), $1-12$.

Klassen, R., Krawchuk, L. \& Rajani, S. (2008). Academic procrastination of undergraduates: Low self-efficacy to self-regulate predicts higher levels of procrastination. Contemporary Educational Psychology, 33, 915-931.

Klassen, R. \& Kuzucu, L. (2009). Academic procrastination and motivation of adolescents in Turkey. Educational Psychology, 29 (1), 69-81.

Klingsieck, K. (2013). Procratination. European Psychologist, 18 (1), 24-34. doi:10.1027/10169040/a000138.

Lam-Figueroa, N., Contreras-Pulache, H., Mori-Quispe, E., Nizama-Valladolid, M., Gutiérrez, C., Hinostroza-Camposano, W. \& Hinostroza-Camposano, W. (2011). Adicción a internet: Desarrollo y validación de un instrumento en escolares adolescentes de Lima, Perú. Revista Peruana de Salud Pública, 28 (3), 462-469.

Mann, L. (2016). Procrastination Revisited: A Commentary. Australian Psychologist, 51 , 47-51. doi:10.1111/ap.12208.

Ndege, W., Mutavi, T., Kokonya, D., Nekesa, V., Musungu, B., Obondo, A. \& Wangari, M. (2015). Social Networks and Students Performance in Secondary Schools: Lessonsforman Open Learning Centre, Kenya. Journal of Education and Practice, 6 (21), 171-178.

Parra, V., Vargas, J., Zamorano, B., Peña, F., Velázquez, Y., Ruiz, L. \& Monreal, O. (2016). Adicción y factores determinantes en el uso problemático del internet, en una muestra de jóvenes universitarios. EDUTEC, Revista Electrónica de Tecnología Educativa, 56, 60-73.

Portellano, J. (2005). Introducción a la neuropsicología. Madrid: Mc Graw Hill.

Quant, D. \& Sánchez, A. (2012). Procrastinación académica: concepto e implicaciones. Revista Vanguardia Psicológica, 3 (1), 45-59.

Raines, J. (2012). The Effect of Online Homework Due Dates on College Student in Elementary Algebra. Journal of Studies in Education, 2 (3), 1-18. doi: http://doi.org/10.5296/jse.v2i3.1704.

Ramos, C. (2015). Los paradigmas de la investigación científica. Avances en Psicología, 23 (1), 9-17.

Ramos, C. \& Pérez-Salas, C. (2015). Relación entre el modelo hibrido de las funciones ejecutivsa y el trastorno por déficit de atención con hiperactividad. Psicología desde el Caribe, 32 (2), 299-314. 
Ramos, C. \& Freire, I. (2016). Prácticas decoloniales en estudiantes universitarios. Quito: Universidad Tecnológica Indoamérica.

Torres-Díaz, J., Duart, J., Gómez-Alvarado, H., Marín-Gutiérrez, I. \& Segarra-Faggioni, V. (2016). Usos de Internet y éxito académico en estudiantes universitarios. Comunicar, 48 (24), 61-70.

Universidad de Navarra. (2012). Cerebro adicto: los efectos de las drogas, el alcohol o internet. Navarra, España: Disponible en: http://www.agenciasinc.es/Noticias/Cerebro-adicto-losefectos-de-las-drogas-el-alcohol-o-internet.

Williams, J. (2008). Revising the declaration oh Helsinki. World medical journal. 54 (4), 120-122.

Wilson, B. \& Bguyen, T. (2012). Belonging to Tomorrow: An Overview of Procrastination. International Journal of Psychological Studies, 4 (1), 211-217.

Xu, Z. (2016). Just Do It! Reducing Academic Procrastination of Secondary Students. Intervention in School and Clinic, 51 (4), 212-219. 
